\title{
The Capacity of a Channel with an Image as the Information Source
}

\author{
Lev B. Levitin, Tommaso Toffoli \\ Department of Electrical \& Computer Engineering, Boston University, Boston, USA \\ Email: levitin@bu.edu, tt@bu.edu
}

Received 18 April 2014; revised 28 May 2014; accepted 22 June 2014

Copyright (C) 2014 by authors and Scientific Research Publishing Inc.

This work is licensed under the Creative Commons Attribution International License (CC BY). http://creativecommons.org/licenses/by/4.0/

(c) (i) Open Access

\begin{abstract}
We consider the physical limitations imposed on the information content of an image by the wave and quantum nature of light, when the image is obtained by illuminating a reflecting or transmitting planar object by natural (i.e., fully thermalized) light, or by observation of an object emitting incoherent (thermal) radiation. The discreteness of the degrees of freedom and the statistical properties of thermal radiation are taken into account. We derive the maximum amount of information that can be retrieved from the object. This amount is always finite and is proportional to the area of the object, the solid angle under which the entrance pupil of the receiver is seen from the object, and the time of observation. An explicit expression for the information in the case where the information recorded by the receiver obeys Planck's spectral distribution is obtained. The amount of information per photon of recorded radiation is a universal numerical constant, independent of the parameters of observation.
\end{abstract}

\section{Keywords}

Quantum Communication, Quantum Limits on Information Retrieval, Information Transmission by Thermal Radiation, Information Content of an Image, Optical Information Channels

\section{Introduction}

A very general and most common way to store information is to encode it in the optical properties of an object. One can then retrieve information by viewing the object by reflected or transmitted light—or even by light emitted by the object itself—for a specified time interval. Such methods, using natural (i.e., thermalized) light, are universally used in photography, television, printing, etc. In the present paper, we discuss the question of what are the fundamental limitations imposed on these methods by the wave and quantum nature of light and by the statistical properties of thermal radiation, and we determine the maximum amount of information that can be 
retrieved from the object by illuminating it in this way and observing its optical image during a read-out window of a prescribed time width. We exclude from consideration cases, like holography, which make use of coherent rather than thermal radiation.

The problem under consideration can be reduced to the evaluation of the capacity of a specific classicalquantum information channel (a channel where classical signals are encoded within quantum states).

Though the bulk of work in this direction was done throughout the last few decades (e.g., [1]-[8]), the special case of thermal radiation deserves a detailed analysis. Note that thermal radiation has maximum entropy for a given average occupation number (the average number of photons per field oscillator); therefore, it is the most "noisy" information carrier.

\section{General Assumptions}

For simplicity, but without loss of generality, let the external surface of the object be a plane of area $S$. The optical properties of this surface as far as reflected light is concerned may be specified by a reflectivity $a(x, y, v)$ - a function of a point $(x, y)$ of the surface and of the frequency $v$ of the radiation. (Here and below we take this to be the reflectivity in the direction to the observer; it is inessential for us whether the reflection is diffuse or specular.) As far as we are only interested in limitations owed to the physical nature of light, we can disregard the material structure of the surface. Even though, for any material, reflectivity is well-defined only for areas that are large with respect to interatomic distances, the idealization of reflectivity as a point function is reasonable when the wavelengths that most significantly contribute to the spectrum of natural light sources are large with respect to interatomic distances.

Similarly, for transmitted light, the function $a(x, y, v)$ should express a transmittance. Finally, in the case of self-luminous surface, $a(x, y, v)$ will be the ratio of the spectral intensity of the radiation at a given point of the surface to the maximum available spectral intensity.

We also assume that a function $a(x, y, v)$ can be assigned independently for the two polarization states of radiation.

\section{Degrees of Freedom of Radiation}

The set of possible functions $a(x, y, v)$ is restricted only by the inequality $0 \leq a(x, y, v) \leq 1$. Obviously, an arbitrarily large amount of information could be encoded in the object by associating with it a function of this kind. However, the use of electromagnetic radiation for reading off information changes the situation in principle, and this is what this paper is about.

Indeed, suppose the area $S$ is viewed by an optical radiation detector whose entrance pupil is seen under a solid angle $\Omega$ from any point of that area. Then, as is well known (see [9]), the number of "spatial” degrees of freedom, or, in other words, the number of field oscillators (or photon quantum states) differing by the direction of their wave vector (or, for another choice of set of oscillators, by the spatial localization of photons) is equal to

$$
G_{s}=\frac{v^{2} \Omega S}{c^{2}}
$$

where $v$ is the frequency of a field oscillator and $c$ the speed of light.

If the object is observed for a time interval $\tau$, then the frequency uncertainty of the photon quantum states is $\Delta v=1 / \tau$, and the number of states of different frequency in a frequency interval $\delta v$ is equal to

$$
G_{f}=\frac{\delta v}{\Delta v}=\tau \delta v .
$$

Taking into account the two possible polarization states, we obtain, for the total number of radiation degrees of freedom,

$$
G=\frac{2}{c^{2}} v^{2} \Omega S \tau \delta v
$$

(under the assumption that $v^{2} \Omega S \tau \delta v / c^{2} \gg 1$, i.e., that the geometric optics approximation is valid); thus, we 
have a finite number of degrees of freedom.

It makes sense to associate a reflectivity $a$ only to object details which do not exceed the space and frequency resolution of the viewer, and thus to areas $\Delta S=c^{2} / v^{2} \Omega$ and frequency intervals $\Delta v=1 / \tau$. Thus, with the present recording/readout method, the retrievable information is specified by the values of a set of $G$ random variables, namely, the reflectivities for each of the field oscillators. This amount of information will be maximum when all those random variables are independent; in that case, it will equal the sum of the amounts of information over all the variables.

\section{Statistics of Radiation and Information}

Even for a single degree of freedom, the amount of information would be infinite if the reflectivity $a$ could take on an infinite number of well-distinguishable values. But such a dream becomes untenable as soon as the quantum nature of light and the statistical properties of thermal radiation come into play.

As is well known (see [10]), in the radiation of thermal sources the states of field oscillators are statistically independent, and described by a Gibbs distribution

$$
p(n)=\frac{1}{\bar{n}+1}\left(\frac{\bar{n}}{\bar{n}+1}\right)^{n},
$$

where $n$ is the occupation number —or the number of photons in a given quantum state-and $\bar{n}$ the mean occupation number.

Thus, if a source of thermal radiation is used as an information transmitter, the expected value $\bar{n}$ (rather than the exact number of photons $n$ ) is the input signal. In other words, the signal is the value of the effective temperature for a given degree of freedom of the electromagnetic field.

A remarkable property of distribution (4) of thermal radiation is its stability with respect to scattering, aperture constraints, losses due to small quantum efficiency of the photodetectors, etc. In particular, if the surface is illuminated by thermal radiation, and thus obeying Gibbs's distribution, the reflected light also has a Gibbs distribution, with the states of field oscillators being independent provided that the illuminating beam is rather wide and has sufficiently large bandwidth. This follows from the transformational properties of the states of the electromagnetic field with respect to a change from a complete set of field oscillators to another complete set (see, e.g., [7]).

Since the density matrix of thermal radiation is diagonal in energy (or occupation number) representation, the optimal strategy to extract maximum information is measuring the energy, or, in other words, counting the number of photons reaching the receiver. This follows from the fact that the radiation quantum state is completely described by distribution (4) (cf. [4]).

Let $P(v)$ be the average energy of a field oscillator of the radiation source of frequency $v$, and let $r(v)$ be the fraction of the energy which is recorded by the detector in the calibration case of reflectivity $a$ equal to unity. Then, assigning a specific value of $a$ means specifying the mean number $\bar{n}$ of recorded photons for each field oscillator, where $\bar{n}=\frac{\operatorname{ar}(v) P(v)}{h v}$.

The maximum mean number of recorded photons will of course be $\bar{n}_{m}=\frac{r(v) P(v)}{h v}$.

As shown in Appendix, when the mean number of photons distributed according to (4) is limited by a maximum value $\bar{n}_{m} \leq 9$ (in the optical range, this corresponds to temperatures $T \leq 3 \times 10^{5}{ }^{\circ} \mathrm{K}$ ), optimal encoding is achieved by using as signal levels just two mean-photon-number values, namely, $\bar{n}=0$ and $\bar{n}=\bar{n}_{m}$ (corresponding to reflectivity values $a=0$ and $a=1$ ). In this case, the maximum amount of information per field oscillator is, in nats,

$$
I_{m}(v)=\ln \left[1+\frac{r(v) P(v)}{r(v) P(v)+h v}\left(\frac{h v}{r(v) P(v)+h v}\right)^{\frac{h v}{r(v) P(v)}}\right]
$$


When a spectral band from $v_{0}$ to $v_{1}$, assuming $\left(v_{1}-v_{0}\right) \tau \gg 1$, with (5) and (7) taken into account, we obtain that the maximin amount of information that can be obtained by observation of an object illuminated by incoherent light is

$$
J_{m}=\frac{2 \tau \Omega S}{c^{2}} \int_{v_{1}}^{v_{2}} v^{2} I_{m}(v) \mathrm{d} v
$$

Thus, the amount of information increases proportionally to the area $S$ of the object and the time $\tau$ of observation.

Then, the capacity of such a channel (the maximum information per unit time) is

$$
C=J_{m} / \tau \text {. }
$$

It will be interesting to derive an explicit expression for the amount of information in the case when the radiation recorded by the detector obeys Planck's spectral distribution (for instance, when the illumination source is a black body of temperature $T$ and $r(v)=1)$, i.e., when

$$
r(v) P(v)=h v /\left(\mathrm{e}^{\frac{h v}{k T}}-1\right)
$$

where $k$ is Boltzmann's constant and $h$ Planck's constant. Let the frequency band be infinite ${ }^{1}$. Then

$$
J_{m}=\frac{2 \tau \Omega S}{c^{2}} \int_{0}^{\infty} v^{2} \ln \left[1+\mathrm{e}^{-\frac{h v}{k T}}\left(1-\mathrm{e}^{-\frac{h v}{k T}}\right)^{\mathrm{e}^{\frac{h v}{k T}}-1}\right] \mathrm{d} v=\frac{2 \tau \Omega S(k T)^{3}}{c^{3} h^{3}} \sigma,
$$

where $\sigma$ is a numerical constant, given by

$$
\sigma=\int_{0}^{\infty} x^{2} \ln \left(1+\mathrm{e}^{-x}\left(1-\mathrm{e}^{-x}\right)^{\mathrm{e}^{x}-1}\right) \mathrm{d} x \approx 0.772 .
$$

The total number of photons recorded by the receiver is in this case

$$
N=\frac{2 \tau \Omega S(k T)^{3}}{c^{3} h^{3}} \eta
$$

where $\eta$ is another constant, namely, $\eta=\int_{0}^{\infty} \frac{x^{2}\left(1-\mathrm{e}^{-x}\right)^{\mathrm{e}^{x}-1}}{\mathrm{e}^{x}-1+\left(1-\mathrm{e}^{-x}\right)^{\mathrm{e}^{\mathrm{x}}}} \mathrm{d} x \approx 0.909$. Thus, the amount of information per photon is

$$
\frac{J_{m}}{N}=\frac{\sigma}{\eta} \approx 0.849 \frac{\text { nats }}{\text { photon }} \approx 1.225 \frac{\text { bits }}{\text { photon }} .
$$

his is a universal constant, as it does not depend on the parameters $\tau, \Omega, S$ of observation.

Now let the solid angle $\Omega$ take on the maximum possible value $2 \pi$. Then the maximum amount $R_{m}$ of information per unit time per unit area is

$$
\begin{aligned}
& R_{m}=\frac{4 \pi(k T)^{3}}{c^{2} h^{3}} \sigma=\frac{\sigma \sqrt{2}}{\pi^{2} \sqrt{c}}\left(\frac{15 P}{\pi h}\right)^{\frac{3}{4}}, \\
& \text { where } P=\frac{4 \pi}{c^{2}} \int_{0}^{\infty} v^{2} \frac{h v}{\mathrm{e}^{\frac{h v}{k T}}-1} \mathrm{~d} v=\frac{4 \pi^{5}(k T)^{4}}{15 c^{2} h^{3}}
\end{aligned}
$$

\footnotetext{
${ }^{1}$ At low frequencies, geometrical optics is not valid, and one cannot use (3); furthermore, for $r(v) P(v) / h v>9$, (5) is not correct. But the contribution of low frequencies is small due to the factor $v^{2}$ in the integrand, and this allows one to extend the range of integration to zero.
} 
is the energy flux of reflected radiation at maximum signal level per unit area.

Thus, the maximum amount of retrieved information grows with the 3/4 power of radiation energy.

\section{References}

[1] Davies, E. (1977) Quantum Communication Systems. IEEE Transactions on Information Theory, 23, 530-534.

[2] Gordon, J. (1962) Quantum Effects in Communications Systems. Proceedings of IRE, 50, 1898-1908.

[3] Lebedev, D.S. and Levitin, L.B. (1966) Information Transmission by Electromagnetic Field. Information and Control, 9, 1-22. http://dx.doi.org/10.1016/S0019-9958(66)90074-X

[4] Levitin, L.B. (1969) On the Quantum Measure of Information. Proceedings of the 4th Conference on Information Theory, Tashkent, 111-116. English Translation in Annales de la Fondation Louis de Broglie, 21, 1996, 345-358.

[5] Levitin, L.B. (1987) Information Theory for Quantum Systems. In: Blaquière, A., Diner, S. and Lochak, G., Eds., Information, Complexity and Control in Quantum Physics, Springer, Berlin, 15-47. http://dx.doi.org/10.1007/978-3-7091-2971-5 2

[6] Levitin, L.B. (1994) Entropy Defect and Information for Two Quantum States. Open Systems and Information Dynamics, 2, 319-329. http://dx.doi.org/10.1007/BF02228857

[7] Mitiugov, V. (1976) Physical Principles of Information Transmission Theory. Sovietskoe Radio, Moscow City (in Russian).

[8] Schumacher, B. and Westmoreland, M. (1997) Sending Classical Information via a Noisy Quantum Channel. Physical Review A, 56, 131-138. http://dx.doi.org/10.1103/PhysRevA.56.131

[9] Gabor, D. (1961) Light and Information. Progress in Optics, 1, 111-152.

[10] Landau, L. and Lifshits, E. (1980) Statistical Physics. 3rd Edition, Butterworth, Heinemann.

[11] Levitin, L.B. (1965) Information Transmission by Thermal Radiation. Proceedings of 2nd National Conferences on Coding Theory and Its Applications, Nauka, Sect. 5, 49-55 (in Russian). 


\section{Appendix}

Let us calculate the maximum information per degree of freedom of radiation. Denote, for convenience,

$$
\frac{\bar{n}}{\bar{n}+1}=x \text { and } \quad \frac{\bar{n}_{m}}{\bar{n}_{m}+1}=x_{m} .
$$

For a given $x$, the distribution of the number of recorded photons is

$$
p(n / x)=(1-x) x^{n} .
$$

Let $f(x)$ the probability density function of the variable $x$. Then the information in the number $n$ of recorded photons about the signal value $x$ is

$$
\begin{aligned}
I & =\sum_{n=0}^{\infty} \int_{0}^{x_{m}} f(x) p(n / x)\left[\ln p(n / x)-\ln \int_{0}^{x_{m}} f(x) p(n / x) \mathrm{d} x\right] \mathrm{d} x \\
& =\int_{0}^{x_{m}} f(x)\left[\ln (1-x)+\frac{x}{1-x} \ln x\right] \mathrm{d} x-\sum_{n=0}^{\infty} \int_{0}^{x_{m}} f(x)(1-x) x^{n} \mathrm{~d} x \ln \int_{0}^{x_{m}} f(x)(1-x) x^{n} \mathrm{~d} x .
\end{aligned}
$$

Variational analysis of the functional $I$ (see [11]) brings up a remarkable result. That is, if $x_{m} \leq 0.9$ (or $\bar{n} \leq 9$ ), the maximun information is achieved for a distribution of the form

$$
f(x)=\left(1-\frac{x_{0}}{x_{m}}\right) \delta(x)+\frac{x_{0}}{x_{m}} \delta\left(x-x_{m}\right)
$$

where

$$
x_{0}=\frac{x_{m}}{\left(1-x_{m}\right)^{\frac{x_{m}}{x_{m}-1}}+x_{m}} .
$$

Thus, if the average occupation numbers are not too large, it is optimal to use only two signal levels, 0 and $x_{m}$. The reason for that effect is the breadth of distribution (19), which makes intermediate values of the signal poorly distinguishable.

From (17)-(19), we obtain that the maximum information per degree of freedom (one field oscillator) is

$$
I_{m}=\ln \left[1+x_{m}\left(1-x_{m}\right)^{\frac{1-x_{m}}{x_{m}}}\right],
$$

corresponding to (5) . 